\title{
Frailty in perioperative patients in three South African academic hospitals
}

\author{
N T N Leopold-George, ${ }^{1}$ MB ChB, DA (SA), FCA (SA); G D Nethathe, ${ }^{2}$ MB ChB, DA (SA), FCA (SA), FANZCA, MMed (Anaes), \\ Cert Critical Care (SA), PGDHSE \\ ${ }^{1}$ Specialist Anaesthesiologist, Private Practice, Johannesburg, South Africa \\ ${ }^{2}$ Division of Critical Care, School of Clinical Medicine, Faculty of Health Sciences, University of the Witwatersrand, Johannesburg, South Africa; \\ and Department of Anaesthesia and Perioperative Medicine, Royal Brisbane and Women's Hospital, Brisbane, Australia
}

Corresponding author: N T N Leopold-George (ngoztonye@yahoo.com)

Background. Frailty is a state characterised by diminished physiological reserve that leaves an individual vulnerable to external stressors and delays recovery. Frailty assessments are proving to be more valuable in predicting poor perioperative outcomes than other well-known perioperative risk assessment tools. Very few studies using validated frailty assessment tools have been done to assess the prevalence of frailty in South Africa (SA), and none have assessed the intraoperative implications of frailty in a surgical population.

Objectives. To determine the demographics and frailty levels of patients presenting for surgery at three academic hospitals in Johannesburg, compare intraoperative complications between the frail and non-frail patients, and compare the association between frailty scores and American Society of Anesthesiologists Physical Status (ASA-PS) scores.

Methods. We prospectively enrolled 299 patients aged 18 - 90 years undergoing various types of elective surgery between mid-November 2016 and mid-March 2017 in three SA academic hospitals. Frailty was assessed using the nine-point Clinical Frailty Scale (CFS) and defined as a score of $\geq 5$. The CFS and demographic and clinical data were documented by the anaesthetists assigned to the respective elective lists. The primary outcome measure was intraoperative complications (hypotension, desaturation, and need for vasopressors and blood transfusion). We also compared associations between the patients' comorbidities and frailty and those between the CFS and ASA-PS scores.

Results. Of a total of 299 patients included in the study (mean age (standard deviation) 50.6 (15.8) years), 156 (52\%) were women and 67 $(22 \%)$ were classified as frail. Compared with patients who were not classified as frail, the frail group had significantly higher incidences of hypotension (odds ratio (OR) 1.87, 95\% confidence interval (CI) $1.083-3.259$; $p=0.02$ ) and desaturation (OR 3.79, 95\% CI 1.367 - 10.54; $p=0.01$ ), and were more likely to need vasopressors (OR 2.81, 95\% CI 1.607 - 4.912; $p=0.00$ ) and blood transfusion (OR 3.26, $95 \%$ CI 1.138 9.368; $p=0.02$ ). On multivariable logistic regression analysis, adjusting for factors related to frailty such as age, gender and comorbidities, desaturation was significantly associated with frailty (adjusted OR (aOR) 4.21, 95\% CI 1.31 - 13.53; $p=0.01$ ), and the frail were more likely to require blood transfusion (aOR 5.36, 95\% CI $1.50-19.16 ; p=0.01$ ) and were older and had more comorbidities. Higher ASA-PS scores were also strongly associated with frailty.

Conclusions. The prevalence of frailty was high among surgical patients. Consistent with other studies, frailty was associated with older age and multiple comorbidities. The association between frailty and intraoperative complications found in this study may indicate and help inform areas of further research.

S Afr Med J 2019;109(7):535-540. DOI:10.7196/SAMJ.2019.v109i7.13439

The ageing population is growing at a rapid rate worldwide, resulting in an increased number of ageing individuals requiring medical services and presenting for surgical procedures. ${ }^{[1]}$ In 2010, twothirds of the world's population aged $>60$ years lived in developing countries, ${ }^{[2]}$ and the World Health Organization (WHO) reports that the number of people aged $>60$ will effectively double by $2050 .{ }^{[3]}$ In the USA, $>40 \%$ of surgical procedures are performed on elderly patients. ${ }^{[4]}$ The increasing number of operations being performed on elderly patients has made it necessary to identify and manage geriatric conditions that place these individuals at increased surgical and anaesthetic risk. ${ }^{[4,5]}$

Frailty is the depletion of physiological and functional reserve across many organ systems, leading to a diminished capacity to withstand environmental stressors such as surgery. ${ }^{[6]}$ An independent predictor of mortality, morbidity, complications and increased length of stay perioperatively, ${ }^{[7]}$ it is likely to become one of the most problematic public health issues and will place a heavy strain on already overburdened healthcare systems. ${ }^{[8]}$ Although often associated with advanced chronological age, frailty can also affect the non-elderly. Rockwood et al. ${ }^{[9]}$ found the prevalence of frailty to be $2.0 \%$ in individuals aged $>30$ years, ${ }^{[9]}$ which is similar to the findings of Kehler et al. ${ }^{[10]}$ that the prevalence of frailty in Canadians between the ages of 18 and 34 was $1.8-5.3 \%$, depending on the frailty assessment used.

A number of assessment methods have been used as indicators of overall health and physiological status. The American Society of Anaesthesiologists Physical Status (ASA-PS) scoring system is a scale from 1 through 6 in increasing order of risk, with an ' $E$ ' to denote emergency surgery, that is assigned to a patient by the anaesthesiologist before surgery. ${ }^{[1]}$ Originally designed to describe the overall health status of a patient, it has since been used as a risk stratification tool and found to be predictive of postoperative morbidity and mortality. ${ }^{[12]}$ The Revised Cardiac Risk Index is a simplified version of an earlier index. ${ }^{[13]}$ It has six predictive factors 
with one point assigned per factor and with an increasing number of points corresponding to increased rates of perioperative cardiac risk. ${ }^{[14]}$ Frailty assessments have been shown to be better predictors of postoperative complications, mortality and morbidity in the aged than other preoperative assessments. ${ }^{[15-17]}$

There are many assessment models that have been used to assess frailty. The two most commonly used models are the frailty phenotype $^{[18]}$ and the frailty index or deficit accumulation. ${ }^{[19]}$ Both models have mainly been used for research purposes, and there have been concerns about their usefulness in a clinical setting. Screening tools such as the Clinical Frailty Scale (CFS) from Dalhousie University in Canada were subsequently developed for practical clinical use. ${ }^{[20]}$ The CFS is a 9-point scale that uses pictographs to depict degrees of functional dependency. The scale ranges from 1 (very fit) to 9 (terminally ill), and a score of $\geq 5$ suggests frailty. Most studies using any one of the validated frailty measuring tools have been conducted in developed countries on predominantly white patients, with few published studies in the developing world and fewer still in Africa. ${ }^{[21]}$ One study utilising data from the WHO study on global ageing and adult health found the prevalence of frailty to be $38 \%$ in South Africa (SA) and $37.9 \%$ in Ghana in adults aged $\geq 50$ years. ${ }^{[2]}$ Another SA study compared frailty between HIV-positive and HIV-negative adults. ${ }^{[23]}$ The mean ages of the HIV-positive and HIV-negative groups were 41.1 years and 42 years, respectively. There was a $19.4 \%$ prevalence of frailty in the HIV-positive adults compared with $13.3 \%$ in those who were HIV-negative.

Combining risk assessment tools such as the ASA-PS and frailty assessments to improve postoperative risk prediction could increase the probability of correct identification of patients who are likely to suffer adverse intraoperative and postoperative adverse events, and has been suggested by some authors. ${ }^{[5,24]}$ However, combinations could make these tools more complex to use and limit their applicability to a wider population, and are likely to require extensive validation studies before they can be put into practice. Although much has been written about the postoperative adverse outcomes and mortality related to frailty, little is known about how frailty impacts on anaesthetic complications intraoperatively, or on the nature of utilisation of health services such as the intensive care unit (ICU) by patients assessed as frail. Correct risk assessment will identify the most vulnerable and may help facilitate discussions about realistic treatment goals.

\section{Objectives}

To determine the prevalence of frailty and assess the intraoperative implications of frailty in a surgical population in three academic hospitals in Johannesburg, SA. We also assessed the association between frailty and comorbid illnesses and the association between the ASA-PS score and the CFS. Finally, we determined whether participants assessed as frail were more likely to go to a high-care unit or an ICU facility postoperatively.

\section{Methods}

Approval for the study was obtained from the Human Research Ethics Committee (Medical) of the University of the Witwatersrand (ref. no. M160756). Participants aged 18 - 90 years presenting for elective surgery at Chris Hani Baragwanath Academic Hospital (CHBAH), Charlotte Maxeke Johannesburg Academic Hospital (CMJAH) and Helen Joseph Hospital (HJH) between mid-November 2016 and mid-March 2017 were included. The study period was effectively 4 months. Combined, these hospitals operate on an average of 2500 elective cases monthly.
This was a prospective, observational and cross-sectional study that employed convenience sampling. The surgical specialties included were general, gynaecological, orthopaedic, maxillofacial, ear, nose and throat, urological, neurosurgical and vascular. We excluded patients known to be pregnant and those with a diagnosis of mental impairment or dementia without any access to family members for information on frailty.

Frailty was defined using the CFS, ${ }^{[20]}$ which is a validated 9-point assessment from the Canadian Study on Health and Aging (Table 1). Patients were defined as frail if they scored $\geq 5$ on the CFS. Copies of the data collection sheet and the CFS were placed in the operating theatre receiving areas at the three sites. Patients presenting for any of the included elective surgeries were identified during the preoperative assessments by the anaesthetists assigned to various elective lists. The anaesthetists included medical officers and registrars at different levels of training. After obtaining written informed consent from the patient or family member, demographic data and clinical data, which included comorbidities and planned procedure, were documented. An ASA-PS score and CFS was assigned on the data collecting sheet. Intraoperative complications and postoperative destination were documented.

All data were entered into a password-protected Microsoft Excel 2016 spreadsheet (Microsoft, USA). Patient-identifiable data were removed. The data were analysed using Stata version 13 (StataCorp, USA). Descriptive statistics were tabulated using means and percentages. The $\chi^{2}$ test and Fisher's exact test were used to describe the association between categorical variables. Logistic regression analysis was used to assess factors associated with frailty. Univariate logistic regression analysis was performed for each of the variables. Multivariable logistic regression analysis was performed to adjust for the possible confounding variables. A $p$-value $<0.05$ was considered statistically significant for all analyses.

\section{Results}

A total of 312 data collection forms were filled in and returned by the attending anaesthetists. We collected data on these 312 patients. Thirteen were excluded in the final analysis ( 7 patients did not undergo the planned surgery, and 6 forms were not filled in and/or consent was not documented), leaving a total of 299 patients. There were 156 women $(52 \%)$ and 143 men $(48 \%)$. The mean (standard deviation) age was 50.6 (15.8) years (range 21 - 90).

The CFSs assigned ranged from 1 (fit) to 8 (very severely frail). Of the patients, 67 (22\%) were classified as frail, with $39(58 \%)$ of these being female and 28 (42\%) male. There were more frail women than men, as shown in Table 2 . This was not statistically significant $(p=0.263)$.

At CHBAH 27 out of 106 patients (25.4\%) were frail, at CMJAH 28 out of $112(25 \%)$ were frail, and at HJH 12 out of 81 (15\%) were frail. Of all the patients, $222(74 \%)$ had an ASA-PS score of 1 or 2 whereas $75(26 \%)$ had an ASA-PS score of 3 or 4 . Higher ASA-PS scores were strongly associated with frailty relative to an ASA-PS score of 1, with an ASA-PS score of 3 being the most highly associated with frailty (odds ratio (OR) 19.01, 95\% confidence interval (CI) 6.870 - 52.614; $p<0.001)$.

Between the ages of 18 and 34 years, $4 \%$ of patients were assessed as frail, in the 35 - 49 age group 13\%, in the 50 - 64 age group $37 \%$, and in the over-65 age group 45\%. Age was significantly associated with frailty (OR 1.06, 95\% CI $1.034-1.084 ; p<0.001$. Frailty was also associated with an increased number of comorbidities. Older patients and those with more comorbidities were also more likely to experience intraoperative complications such as hypotension and need for vasopressors (Table 3). 
Table 1. Clinical Frailty Scale ${ }^{[20]}$

\begin{tabular}{ll}
\hline 1. Very fit & Robust, active, energetic and motivated. Commonly exercise. Fittest for their age \\
2. Well & No active disease symptoms, but less fit than category 1 \\
3. Managing well & Medical problems are well controlled, but not regularly active \\
4. Vulnerable & Not dependent on others for daily help. Symptoms limit activities \\
5. Mildly frail & More evident slowing. Need help in higher-order instrumental activities of daily living \\
6. Moderately frail & Need help with all outside activities. Need help with instrumental and non-instrumental activities of daily living \\
7. Severely frail & Completely dependent for personal care. Not at high risk of dying \\
8. Very severely frail & Completely dependent. Approaching the end of life \\
9. Terminally ill & Approaching the end of life, or life expectancy $<6$ months in those who are not otherwise evidently frail
\end{tabular}

Table 2. Baseline characteristics of study participants

\begin{tabular}{|c|c|c|c|}
\hline & $n(\%)$ & Age (years), mean (SD) & Gender, $\boldsymbol{n}(\%)$ \\
\hline \multirow[t]{2}{*}{ Overall } & 299 & $50.6(15.8)$ & Women 156 (52.2) \\
\hline & & & Men 143 (47.8) \\
\hline \multirow[t]{2}{*}{ Frail $(\mathrm{CFS} \geq 5)$} & $67(22.4)$ & $62.29(15.45)$ & Women 39/67 (58.2) \\
\hline & & & Men 28/67 (41.8) \\
\hline \multirow[t]{2}{*}{ Non-frail } & $232(77.6)$ & $47.30(14.32)$ & Women 117/232 (50.4) \\
\hline & & & Men 115/232 (49.6) \\
\hline
\end{tabular}

Before adjusting for confounding variables, frailty was significantly associated with an increased incidence of hypotension and desaturation, need for blood transfusion and need for vasopressors. We then adjusted for confounding variables (age, gender, comorbidities) that are related to frailty and intraoperative complications. After adjustment, desaturation was significantly associated with frailty (adjusted odds ratio (aOR) 4.12, 95\% CI $1.202-14.139 ; p=0.024$ ) as well as need for blood transfusion (aOR 5.36, 95\% CI 1.50 - 19.16; $p=0.01$ ), as shown in Table 3. Age, gender and comorbidities were not significantly related to frailty in either univariate or multivariable logistic regression analysis when assessing desaturation and need for blood transfusion.

Patients assessed as frail also had an increased likelihood of going to high care postoperatively (OR 3.01, 95\% CI $1.073-8.447$; $p=0.036$ ).

\section{Discussion}

The prevalence of frailty is said to be higher in developing countries than in developed countries, where it ranges from $5.4 \%$ to $44 \%$ in community-dwelling elderly ${ }^{[2]}$ and is reported to be between $4.1 \%$ and $50.3 \%$ in patients of all ages presenting for surgical procedures, depending on the assessment tool used and the population sampled. ${ }^{[25]}$ The present study found that $22 \%$ of patients undergoing elective surgery in three large academic hospitals in Johannesburg were frail. This percentage is lower than our estimate of $30 \%$ and is also lower than the reported rates of frailty among the hospitalised elderly. ${ }^{[4,15]}$ Our study, however, was one of the few that included age groups $\geq 18$ years, and on further analysis it was found that $45 \%$ of patients aged $>65$ were frail, which is on the higher end of prevalence rates generally reported for surgical patients. The distribution of frailty according to type of surgery is shown in Table 4.

Other studies have used the CFS to predict mortality and adverse outcomes. Bagshaw et al. ${ }^{[26]}$ used the CFS in critically ill adults aged $\geq 50$ years and showed a prevalence of frailty of $32.8 \%$. They also showed that in-hospital mortality was higher in the frail (32\% v. $16 \%$ in the non-frail) and that major adverse events were more common (39\% v. $29 \%$ ). Similar to our study, the frail were older and had more comorbidities, which is also consistent with many other studies. ${ }^{[18,27,28]}$
Hewitt et al. ${ }^{[29]}$ showed that $28 \%$ of patients aged $\geq 65$ in three acute surgical admission units were frail according to the CFS. The frail individuals spent longer in hospital and were more likely to die within 30 and 90 days after surgery.

The present study also demonstrates the ability of the CFS to predict outcomes even when used by clinicians without prior training on how to use the scale. In a comparable study, the CFS was used by junior doctors without any prior training in patients aged $>65$ years admitted to an acute general medical unit. ${ }^{[30]}$ In this study, frailty was associated with functional decline $(p=0.011)$ and mortality within 3 months $(p=0.012)$.

There are no current studies with which we can directly compare our study, as most studies have focused on postoperative outcomes. ${ }^{[31]}$ We could not find any studies that aimed to associate frailty with intraoperative complications, although a number of studies have shown a relationship between intraoperative complications and postoperative outcomes. The need for intraoperative blood transfusion was shown to be associated with increased morbidity and mortality in anaemic surgical patients. ${ }^{[32]}$ Intraoperative hypotension was demonstrated to be associated with myocardial injury, acute kidney injury and death. ${ }^{[33]}$

The present study identifies the intraoperative environment as one associated with a high risk of adverse outcomes in the frail. These increased adverse outcomes in the frail should ultimately emphasise the need for intraoperative vigilance and highlight the significance of frailty for all perioperative physicians, including the anaesthetist and not just the surgeon or intensivist.

Univariate logistic regression analysis showed that frailty was associated with an increased risk of intraoperative complications such as hypotension, desaturation, need for vasopressors and blood transfusion. Frailty was only significantly associated with desaturation and need for blood transfusion after multivariable logistic regression analysis, which is possibly a function of a small sample size, with fewer frail patients in the sample than anticipated. Patients with higher levels of frailty on the CFS were more likely to receive an ASA-PS score of $\geq 3$, which is in keeping with the findings of Robinson et al. ${ }^{[34]}$ There was an association between frailty and 


\section{Table 3. Effect of frailty on intraoperative complications}

\begin{tabular}{|c|c|c|c|c|c|c|}
\hline \multirow[b]{2}{*}{ Factors } & \multicolumn{3}{|c|}{$\begin{array}{c}\text { Univariate logistic model to assess } \\
\text { effect of frailty }\end{array}$} & \multicolumn{3}{|c|}{$\begin{array}{l}\text { Multivariable logistic regression controlling for other } \\
\text { variables on effect of frailty on complications }\end{array}$} \\
\hline & OR & $95 \% \mathrm{CI}$ & $p$-value & aOR & 95\% CI & $p$-value \\
\hline \multicolumn{7}{|c|}{ Association between frailty and hypothermia } \\
\hline \multicolumn{7}{|l|}{ Frailty } \\
\hline No & 1 & & & & & \\
\hline Yes & 2.08 & $0.99-4.36$ & 0.051 & - & - & - \\
\hline \multicolumn{7}{|c|}{ Association between frailty and hypotension } \\
\hline \multicolumn{7}{|l|}{ Frailty } \\
\hline No & 1 & & & & & \\
\hline Yes & 1.87 & $1.08-3.25$ & 0.025 & 1.87 & $0.81-4.34$ & 0.14 \\
\hline \multicolumn{7}{|c|}{ Age category (years) } \\
\hline $18-34$ & 1 & & & & & \\
\hline $35-49$ & 3.04 & $1.23-7.49$ & 0.01 & 0.80 & $0.24-2.68$ & 0.72 \\
\hline $50-64$ & 4.38 & $1.77-10.81$ & 0.001 & 1.76 & $0.54-5.70$ & 0.34 \\
\hline$\geq 65$ & 8.0 & $3.06-20.87$ & 0.000 & 1.65 & $0.44-6.16$ & 0.45 \\
\hline \multicolumn{7}{|l|}{ Sex } \\
\hline Female & 1 & & & & & \\
\hline Male & 0.81 & $0.509-1.300$ & 0.394 & - & - & - \\
\hline \multicolumn{7}{|c|}{ Comorbidities, $n$} \\
\hline 0 & 1 & & & & & \\
\hline 1 & 1.94 & $1.08-3.50$ & 0.02 & 0.77 & $0.31-1.90$ & 0.57 \\
\hline 2 & 2.67 & $1.33-5.34$ & 0.005 & 0.74 & $0.25-2.16$ & 0.58 \\
\hline 3 & 1.84 & $0.78-4.35$ & 0.16 & 0.64 & $0.17-2.38$ & 0.51 \\
\hline 4 & 1.10 & $0.20-6.06$ & 0.90 & - & - & - \\
\hline \multicolumn{7}{|c|}{ Association between frailty and desaturation } \\
\hline \multicolumn{7}{|l|}{ Frailty } \\
\hline No & 1 & & & & & \\
\hline Yes & 3.79 & $1.36-10.54$ & 0.01 & 4.21 & $1.31-13.53$ & 0.01 \\
\hline \multicolumn{7}{|c|}{ Association between frailty and need for blood transfusion } \\
\hline \multicolumn{7}{|l|}{ Frailty } \\
\hline No & 1 & & & & & \\
\hline Yes & 3.26 & $1.13-9.36$ & 0.02 & 5.36 & $1.50-19.16$ & 0.01 \\
\hline \multicolumn{7}{|c|}{ Association between frailty and arrhythmia } \\
\hline \multicolumn{7}{|l|}{ Frailty } \\
\hline No & 1 & & & & & \\
\hline Yes & 2.34 & $0.38-14.35$ & 0.35 & - & - & - \\
\hline \multicolumn{7}{|c|}{ Association between frailty and need for vasopressors } \\
\hline \multicolumn{7}{|l|}{ Frailty } \\
\hline No & 1 & & & & & \\
\hline Yes & 2.81 & $1.60-4.91$ & 0.00 & 1.57 & $0.83-2.98$ & 0.16 \\
\hline \multicolumn{7}{|c|}{ Age category (years) } \\
\hline $18-34$ & 1 & & & & & \\
\hline $35-49$ & 1.76 & $0.70-4.46$ & 0.22 & 1.67 & $0.64-4.31$ & 0.28 \\
\hline $50-64$ & 4.18 & $1.69-10.33$ & 0.002 & 3.24 & $1.24-8.47$ & 0.01 \\
\hline$\geq 65$ & 8 & $3.06-20.87$ & 0.00 & 6.23 & $2.17-17.82$ & 0.001 \\
\hline Sex & & & & & & \\
\hline Female & 1 & & & & & \\
\hline Male & 0.79 & $0.48-1.28$ & 0.34 & 1.03 & $0.60-1.79$ & 0.89 \\
\hline Comorbid & & & & & & \\
\hline 0 & 1 & & & & & \\
\hline 1 & 1.63 & $0.87-3.84$ & 0.12 & 1.05 & $0.52-2.09$ & 0.88 \\
\hline 2 & 3.33 & $1.63-3.77$ & 0.00 & 1.74 & $0.77-3.91$ & 0.18 \\
\hline 3 & 3.02 & $1.27-7.14$ & 0.01 & 1.74 & $0.66-4.58$ & 0.26 \\
\hline 4 & 1.38 & $0.25-7.61$ & 0.71 & 0.41 & $0.06-2.58$ & 0.34 \\
\hline
\end{tabular}




\begin{tabular}{llll} 
Table 4. Distribution of frailty & & & \\
\hline Type of surgery & Frail, $\boldsymbol{n}(\%)$ & Non-frail, $\boldsymbol{n}(\%)$ & Total, $\boldsymbol{n}(\%)$ \\
\hline Orthopaedic & $44(65.7)$ & $56(24.1)$ & $100(33.4)$ \\
General surgery & $9(13.4)$ & $73(31.5)$ & $82(27.4)$ \\
Urology & $5(7.5)$ & $33(14.2)$ & $38(12.7)$ \\
Gynaecology & 0 & $30(12.9)$ & $30(10.0)$ \\
Neurosurgery & $3(4.5)$ & $9(3.9)$ & $12(4.0)$ \\
Ear, nose and throat & $2(3.0)$ & $10(4.3)$ & $12(4.0)$ \\
Vascular & $4(6.0)$ & $6(2.6)$ & $10(3.3)$ \\
Plastics & 0 & $8(3.4)$ & $8(2.7)$ \\
Maxillofacial & 0 & $6(2.6)$ & $6(2.0)$ \\
Not specified & 0 & $1(0.4)$ & $1(0.3)$ \\
Total & $67(100)$ & $232(100)$ & $299(100)$
\end{tabular}

postoperative destination, with the frail being more likely to go to a high-care unit, but there was no significant association between frailty and ICU admission owing to sample size. The clinical significance of this is unclear.

Although the prevalence of frailty is highest among the elderly, there is a noteworthy prevalence among younger age groups. This prevalence could increase with the increase in communicable and noncommunicable diseases such as HIV, tuberculosis and traumatic injury.

\section{Study strengths and limitations}

A strength of the study is that it is the only study of its kind to be conducted in SA looking specifically at the effect of frailty on intraoperative complications. The study also included patients from a few of the largest hospitals in sub-Saharan Africa. The patients were largely from a low socioeconomic group and were representative of the patient population that is seen at most SA hospitals, as only a small proportion of the SA population can afford private hospital care.

Limitations include the fact that assessment of frailty in a perioperative setting is confounded by the effects of the presenting surgical condition. To minimise the effects of acute surgical conditions, only patients presenting for elective procedures were included.

Data were documented by different anaesthesia providers at different levels of anaesthetic training, and inter-observer reliability was not assessed. It was, however, important to demonstrate the utility of the CFS as a screening tool in a real-life clinical setting where it was intended for use by non-geriatricians. The CFS has shown good interobserver reliability in a previous study. ${ }^{[35]}$

This was a single-centre study with a relatively small sample size. Future larger studies may focus on the association between frailty and postoperative complications, morbidity, length of stay and mortality in an SA surgical population.

\section{Conclusions}

The prevalence of frailty was high among surgical patients. Consistent with other studies, frailty was associated with older age and multiple comorbidities. The association between frailty and intraoperative complications found in this study may indicate and help inform areas of further research.

\section{Declaration. None.}

Acknowledgements. We thank the departments of anaesthesiology at CHBAH, CMJAH and HJH, and Dr Innocent Maposa (biostatistician and senior lecturer in the University of the Witwatersrand School of Public Health) for his assistance with statistical calculations.
Author contributions. Research idea conception: GDN and NTNL-G; data collection: NTNL-G; proposal write-up: NTNL-G and GDN; article writeup: NTNL-G and GDN.

Funding. None.

Conflicts of interest. None.

1. Barnett SR. Perioperative frailty. Adv Anesth 2014;1(32):119-131. https://doi.org/10.1016/ aan.2014.08.012

2. Nguyen TN, Cumming RG, Hilmer SN. A review of frailty in developing countries. J Nutr Health Aging 2015;19(9):941-946. https://doi.org/10.1007/s12603-015-0503-2

3. World Health Organization. World Report on Ageing and Health. Geneva: WHO, 2015.

4. Amrock LG, Deiner S. Perioperative frailty. Int Anesthesiol Clin 2014;52(4):26-41. https://doi. org/10.1097/AIA.0000000000000026

Griffiths R, Mehta M. Frailty and anaesthesia: What we need to know. Contin Educ Anaesth Crit Care Pain 2014;14(6):273-277. https://doi.org/10.1093/bjaceaccp/mkt069

6. Moorhouse P, Rockwood K. Frailty and its quantitative clinical evaluation. J R Coll Physicians Edinb 2012;42(4):333-340. https:///doi.org/10.4997/JRCPE.2012.412
Moord

2012;42(4):333-340. https://doi.org/10.4997//RCPE.2012.412 Beggs T, Sepehri A, Szwajcer A, Tangri N, Arora RC. Frailty and perioperative outcones:
review. Can J Anaesth 2015;62(2):143-157. https://doi.org/10.1007/s12630-014-0273-z

review. Can J Anaesth 2015;62(2):143-157. https://doi.org/10.1007/s12630-014-0273-Z
8. Dent E, Chapman I, Howell S, Piantadosi C, Visvanathan R. Frailty and functional decline indices Dent E, Chapman I, Howell S, Piantadosi C, Visvanathan R. Frailty and functional decline indices
predict poor outcomes in hospitalised older people. Age Ageing 2014;43(4):477-484. https://doi. org/10.1093/ageing/aft181

9. Rockwood K, Song X, Mitnitski A. Changes in relative fitness and frailty across the adult lifespan Evidence from the Canadian National Population Health Survey. CMAJ 2011;183(8):E487-E494. https://doi.org/10.1503/cmaj.101271

0. Kehler DS, Ferguson T, Stammers AN, et al. Prevalence of frailty in Canadians 18 - 79 years old in the Canadian Health Measures Survey. BMC Geriatr 2017;17(1):28. https://doi.org/10.1186/s12877. 017-0423-6

11. Saklad M. Grading of patients for surgical procedures. Anesthesiology 1941;2:281-284.

12. Hopkins TJ, Raghunathan K, Barbeito A, et al. Associations between ASA Physical Status and postoperative mortality at $48 \mathrm{~h}$ : A contemporary dataset analysis compared to a historical cohort. Perioper Med (Lond) 2016;5:29. https://doi.org/10.1186/s13741-016-0054-z

13. Goldman L, Caldera DL, Southwick FS, et al. Cardiac risk factors and complications in non-cardiac surgery. Medicine (Baltimore) 1978;57(4):357-370.

14. Davis C, Tait G, Carroll J, Wijeysundera DN, Beattie WS. The Revised Cardiac Risk Index in the
surgery. Medicine (Baltimore) $978 ; 57$ (4):357-370. Davis C, Tait G, Carroll J, Wijeysundera DN, Beattie WS. The Revised Cardiac Risk Index in the
new millennium: A single-centre prospective cohort re-evaluation of the original variables in 9,519 consecutive elective surgical patients. Can J Anaesth 2013;60(9):855-863. https://doi.org/10.1007/ s12630-013-9988-5

5. Makary MA, Segev DL, Pronovost PJ, et al. Frailty as a predictor of surgical outcomes in older patients. J Am Coll Surg 2010;210(6):901-908. https://doi.org/10.1016/j.jamcollsurg.2010.01.028

6. Farhat JS, Velanovich V, Falvo AJ, et al. Are the frail destined to fail? Frailty index as predictor of surgical morbidity and mortality in the elderly. J Trauma Acute Care Surg 2012;72(6):1526-1530; discussion 1530-1531. https://doi.org/10.1097/TA.0b013e3182542fab

17. Joseph B, Zangbar B, Pandit V, et al. Emergency general surgery in the elderly: Too old or too frail? J Am Coll Surg 2016;222(5):805-813. https://doi.org/10.1016/j.jamcollsurg.2016.01.063

18. Fried LP, Tangen CM, Walston J, et al. Frailty in older adults: Evidence for a phenotype. J Gerontol A Biol Sci Med Sci 2001;56(3):M146-M156. https://doi.org/10.1093/gerona/56.3.M146

19. Mitnitski AB, Mogilner AJ, Rockwood K. Accumulation of deficits as a proxy measure of aging. Sci Mitnitski AB, Mogilner A,, Rockwood K. Accumulation of defcild
World J 2001;1:323-336. https://doi.org/10.1100/tsw.2001.58

20. Rockwood K, Song X, MacKnight C, et al. A global clinical measure of fitness and frailty in elderly . Rockwood K, Song X, MacKnight C, et al. A global clinical measure of
people. CMAJ 2005;173(5):489-495. https://doi.org/ $10.1503 / \mathrm{cmaj} .050051$

21. Cesari M, Prince M, Thiyagarajan JA, et al. Frailty: An emerging public health priority. J Post Acute Long Term Care Med 2016;17(3):188-192. https://doi.org/10.1016/j.jamda.2015.12.016

22. Biritwum RB, Minicuci N, Yawson AE, et al. Prevalence of and factors associated with frailty and disability in older adults from China, Ghana, India, Mexico, Russia and South Africa. Maturitas 2016;91:8-18. https://doi.org/10.1016/j.maturitas.2016.05.012

3. Pathai S, Gilbert C, Weiss HA, et al. Frailty in HIV-infected adults in South Africa. J Acquir Immune Defic Syndr 2013;62(1):43-51. https://doi.org/10.1097\%2FQAI.0b013e318273b631

24. Revenig LM, Canter DJ, Kim S, et al. Report of a simplified frailty score predictive of shortterm postoperative morbidity and mortality. J Am Coll Surg 2015;220(5):904-911.el. https://doi. org/10.1016/j.jamcollsurg.2015.01.053

25. Partridge JS, Harari D, Dhesi JK. Frailty in the older surgical patient: A review. Age Ageing 2012;41(2):142-147. https://doi.org/10.1093/ageing/afr182

26. Bagshaw SM, Stelfox HT, McDermid RC, et al. Association between frailty and short- and longterm outcomes among critically ill patients: A multicentre prospective cohort study. CMAJ 2014;186(2):E95-E102. https://doi.org/10.1503/cmaj.130639 
27. Woods NF, LaCroix AZ, Gray SL, et al. Frailty: Emergence and consequences in women aged 65 and older in the Women's Health Initiative Observational Study. J Am Geriatr Soc 2005;53(8):1321-1330. https://doi.org/10.1111/.j.1532-5415.2005.53405.x

28. Gu D, Dupre ME, Sautter I, Zhu H, Liu Y, Yi Z. Frailty and mortality among Chinese at advanced ages. J Gerontol B Psychol Sci Soc Sci 2009;64(2):279-289. https://doi.org/10.1093/geronb/gbn009

29. Hewitt J, Moug SJ, Middleton M, et al. Prevalence of frailty and its association with mortality in general

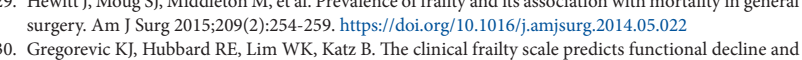
30. Gregorevic KJ, Hubbard RE, Lim WK, Katz B. The clinical frailty scale predicts functional decline and
mortality when used by junior medical staff: A prospective cohort study. BMC Geriatr 2016;16:117. mortality when used by junior medical staff: A prospective cohort study. BMC Geriatr 2016;16:117.
https://doi.org/10.1186/s12877-016-0292-4 https://doi.org/10.1186/s12877-016-0292-4

31. Basic D, Shanley C. Frailty in an older inpatient population: Using the clinical frailty scale to predic patient outcomes. J Aging Health 2015;27(4):670-685. https://doi.org/10.1177/0898264314558202

32. Glance LG, Dick AW, Mukamel DB, et al. Association between intraoperative blood transfusion and mortality and morbidity in patients undergoing noncardiac surgery. J Am Soc Anesthesiol 2011;114(2):283-292. https://doi.org/10.1097/ALN.0b013e3182054d06
33. Walsh M, Devereaux PJ, Garg AX, et al. Relationship between intraoperative mean arterial pressure and clinical outcomes after noncardiac surgery toward an empirical definition of hypotension. J Am Soc Anesthesiol 2013;119(3):507-515. https://doi.org/10.1097/ALN.0b013e3182a10e26

34. Robinson TN, Wu DS, Pointer L, Dunn CL, Cleveland JC Jr, Moss M. Simple frailty score predicts postoperative complications across surgical specialties. Am J Surg 2013;206(4):544-550. https://doi. postoperative complications across
org/10.1016/j.amjsurg.2013.03.012

35. Grossman D, Rootenberg M, Perri GA, et al. Enhancing communication in end-of-life care: A clinical tool translating between the Clinical Frailty Scale and the Palliative Performance Scale. J Am Geriatr Soc 2014;62(8):1562-1567. https://doi.org/10.1111/jgs. 12926

Accepted 7 January 2019. 